\title{
Multidimensional virtual reality-MVR method: a new method of visualization of multidimensional worlds
}

\author{
Dariusz Jamróz ${ }^{1}$ (1)
}

Published online: 24 April 2019

(c) The Author(s) 2019

\begin{abstract}
The paper presents a new, original method of multidimensional worlds' visualization. It allows to present views of any dimension objects out of which it is possible to construct even the most complicated multidimensional virtual world on a computer screen. Due to this, it is possible to observe multidimensional worlds modeled in this way, analyze mutual relations between multidimensional objects, move between them and, most importantly, verify whether human brain is able to adapt to the perception of more than three-dimensional space. This paper presents example interior views of four-dimensional and fivedimensional labyrinths. It also presents results of the research performed on 97 IT students at the AGH University of Science and Technology. Students in total made 357 attempts to leave virtual four-dimensional and five-dimensional labyrinths, each having three difficulty levels. The method presented in this paper is sufficiently general to allow observation of objects in an $n$-dimensional space for any $n \geq 3$. Simultaneously, it is the natural extension of our reality perception because using this method for $n=3$ we obtain views known to us from our human experience from the three-dimensional space.
\end{abstract}

Keywords Multidimensional virtual reality · MVR method - Multidimensional world · Multidimensional labyrinth . Multidimensional visualization $\cdot$ Perception

\section{Introduction}

The author introduced the notion of the multidimensional virtual reality as a result of the previous research [6]. However, the conducted research was stopped for two reasons. The first one concerned the calculation power necessary to obtain multidimensional worlds' views. Because it is difficult to move around the virtual world if, despite the use of GPU, you need to wait for over a second to obtain each view [6]. The use of the latest GPU accelerators for calculations contributed to solving the above-mentioned problem. The second reason for stopping the research was the lack of possibility to perceive movement forward and backward, which made it difficult to properly perceive the location in the virtual multidimensional

Electronic supplementary material The online version of this article (https://doi.org/10.1007/s00371-019-01653-2) contains

supplementary material, which is available to authorized users.

\section{Dariusz Jamróz}

jamroz@agh.edu.pl

1 Department of Applied Computer Science, AGH University of Science and Technology, al. Mickiewicza 30, 30-059 Kraków, Poland world. The cause was the lack of changes in multidimensional objects' views during approaching and moving away from them by a virtual observer. A new method of visualization presented in this paper is the solution to this problem. By introducing the notion of perspective in multidimensional space, observation of changes in multidimensional objects' views is possible while approaching them and moving away from them.

\section{Related studies}

Other methods found in the literature applied to visualize multidimensional objects did not provide such possibilities as the one presented in this paper; however, they will be mentioned as those which are as close as possible to the conducted research. Four-dimensional blocks in the form of wire frames are presented in paper [15]. Three-dimensional stereoscopic movies of the three-dimensional orthogonal and perspective-based projections of four-dimensional hyperobjects rotating in a four-dimensional space were generated using a plotter. Another approach was to present images of a four-dimensional block and a four-dimensional sphere pre- 
sented in the form of a set of three-dimensional tori [1,3]. Using the next method, four-dimensional objects in the form of three-dimensional slices were presented [1]. Also, the scan-plane conversion to render four-dimensional objects into three-dimensional voxel fields was used for the visualization $[2,16]$. Those fields were then projected onto a two-dimensional screen. Attempts to render points, curves and surfaces in four dimensions were also made [4].

Apart from the approaches allowing to visualize simple multidimensional objects presented above, the literature lacks any up-to-date studies allowing to visualize multidimensional reality comprising many continuous objects (solids), which are additionally observed from the firstperson perspective. Thus, there are no studies in the literature similar to those presented in the paper presenting people's abilities to intentionally move around in such a space. The method presented in the paper is a pioneer solution. The only exception is the previous version of the method [6] (without perspective) introduced by the author, and basically the results obtained in this paper can only be compared with this method. However, in the case of this previous method (without perspective), extensive studies determining the people's abilities to intentionally move around the multidimensional space were abandoned. This resulted from the fact that in the case of several initially examined people, none of them (apart from the author) was able to leave a labyrinth, which was also easier than the one identified in this paper as difficulty level 1 , within a reasonable time frame. Based on the unique results obtained in the paper, it can be thus concluded that the method presented in the paper is a completely new approach and significant contribution to the current state of knowledge.

Visualization of multidimensional spaces is also used during the analysis of multidimensional data sets $[5,7-14,17]$. However, such visualization concerns objects of a completely different type-sets of points located in multidimensional spaces; therefore, we only mention it here.

\section{The MVR method: the method of visualization of multidimensional worlds}

Multidimensional reality goes beyond our human experience. Therefore, while constructing the method for such a space visualization, mathematical tools which will be used should be defined:

Definition 1 The observed space $X$ is defined as any vector space, over an $F$ field of real numbers, $n$-dimensional, $n \geq 3$, with a scalar product.
Definition 2 Let $p_{1}, p_{2} \in X$ be linearly independent, $w \in$ $X$. An observational plane $P \subset X$ is defined as:

$P=\delta\left(w,\left\{p_{1}, p_{2}\right\}\right)$

where

$\delta\left(w,\left\{p_{1}, p_{2}\right\}\right) \stackrel{\text { def }}{=}\left\{x \in X: \exists \beta_{1}, \beta_{2} \in F\right.$

$$
\text { such that } \left.x=w+\beta_{1} p_{1}+\beta_{2} p_{2}\right\}
$$

Observational plane $P$ defined in this way will serve as a screen on which we will present views of objects located in multidimensional observed space $X$. Vector $w$ will indicate the position of the screen center, whereas $p_{1}, p_{2}$ will indicate its axes. In a three-dimensional space, only one line orthogonal to $P$ goes through a given point of observational plane $P$. In spaces with a greater number of dimensions, there is a whole family of such lines. Therefore, to analyze the light rays' course, we have to determine the specific direction in which such rays travel:

Definition 3 The direction of projection $r$ onto the observational plane $P=\delta\left(w,\left\{p_{1}, p_{2}\right\}\right)$ is defined as any vector $r \in X$ if vectors $\left\{p_{1}, p_{2}, r\right\}$ are an orthogonal system.

Definition 4 The following set is called half-space $Z_{(s, d)}$ anchored in $s \in X$ and directed toward $d \in X$ :

$Z_{(s, d)} \stackrel{\text { def }}{=}\{x \in X:(x-s, d) \geq 0\}$

Definition 5 The following set is called the convex solid $Y$ generated by half-spaces $Z_{\left(s_{1}, d_{1}\right)}, Z_{\left(s_{2}, d_{2}\right)}, \ldots, Z_{\left(s_{k}, d_{k}\right)}$ :

$Y \stackrel{\text { def }}{=}\left\{x \in X: x \in \bigcap_{i=1}^{k} Z_{\left(s_{i}, d_{i}\right)}\right\}$

Definition 6 The following set is called the hypersurface $S_{(s, d)}$ anchored in $s \in X$ and directed toward $d \in X$ :

$S_{(s, d)} \stackrel{\text { def }}{=}\{x \in X:(x-s, d)=0\}$

Definition 7 Let $Y$ be a convex solid generated by halfspaces $Z_{\left(s_{i}, d_{i}\right)}$, where $i=1,2, \ldots, k$. The fragment of hypersurface $S_{\left(s_{i}, d_{i}\right)}$ contained in $Y$ is called the face of convex solid $Y$.

Definition 8 We will call multidimensional virtual reality $V R$ consisting of convex solids $Y_{1}, Y_{2}, \ldots, Y_{m}$ :

$\mathrm{VR} \stackrel{\text { def }}{=}\left\{x \in X: x \in \bigcup_{i=1}^{m} Y_{i}\right\}$ 
In this way, we can approximate any multidimensional world comprising continuous objects by an accordingly large number of convex solids.

Let us consider the way light is reflected from the face of multidimensional convex solid $Y$. Let us assume that the dimension of the observed space $X$ and the dimension of convex solid $Y$ are equal to 7 . In this case, the faces of convex solid $Y$ separating its interior from its exterior will be sixdimensional objects. It should be noted that light could be reflected by any point on this face, i.e., in this hypothetical situation by any point belonging to the six-dimensional object (including also the "interior" of such an object, being the face). The question is how bright the light reflected by a point on the face, e.g., of a six-dimensional object, should be. Due to the above definition of hypersurface $S_{(s, d)}$ containing the face, the answer to this question is simple: Brightness depends on direction $d$ of hypersurface $S_{(s, d)}$ containing the face, on the direction of projection $r$, on the light source direction and on the perspective coefficient.

Definition 9 The brightness level of a hypersurface $S_{(s, d)}$ fragment observed from a location with coordinates $\beta_{1}, \beta_{2}$ specifying the location on observational plane $P$ is equal to:

$\mathrm{LUM}=\frac{\left(\xi r-\beta_{1} p_{1}-\beta_{2} p_{2}, d\right)}{\sqrt{\left(\xi r-\beta_{1} p_{1}-\beta_{2} p_{2}, \xi r-\beta_{1} p_{1}-\beta_{2} p_{2}\right)}}$

where

$\beta_{1}, \beta_{2} \in F$-coordinates specifying the location on observational plane $P=\delta\left(w,\left\{p_{1}, p_{2}\right\}\right)$,

$p_{1}, p_{2} \in X$-axes specifying observational plane $P=$ $\delta\left(w,\left\{p_{1}, p_{2}\right\}\right)$

$r \in X$-the direction of projection onto observational plane $P$,

$d \in X$-the vector specifying hypersurface $S_{(s, d)} \subset X$ anchored in $s$ and directed toward $d$,

$\xi \in(0, \infty)$ - the coefficient specifying the perspective degree.

Let us consider another problem. Let us take any location on observational plane $P=\delta\left(w,\left\{p_{1}, p_{2}\right\}\right)$. In order to determine which of the convex solid $Y$ faces is visible from this position on observational plane $P$, we have to know which one of them is the closest. To do this, we have to introduce the notion of distance.

Definition 10 The distance of a hypersurface $S_{(s, d)}$ fragment observed from a location with coordinates $\beta_{1}, \beta_{2}$ specifying the location on observational plane $P$ is equal to:

$\psi=\frac{(s-w-\xi r, d)}{\left(\beta_{1} p_{1}+\beta_{2} p_{2}-\xi r, d\right)}-1$

where $\beta_{1}, \beta_{2} \in F$-coordinates specifying the location on observational plane $P=\delta\left(w,\left\{p_{1}, p_{2}\right\}\right)$,

$p_{1}, p_{2} \in X$-axes specifying observational plane $P=$ $\delta\left(w,\left\{p_{1}, p_{2}\right\}\right)$,

$w \in X$ - the vector specifying the location of the observational plane $P=\delta\left(w,\left\{p_{1}, p_{2}\right\}\right)$ center,

$r \in X$-the direction of projection onto observational plane $P$,

$s, d \in X$-vectors specifying hypersurface $S_{(s, d)} \subset X$ anchored in $s$ and directed toward $d$,

$\xi \in(0, \infty)$ - the coefficient specifying the perspective degree.

The distance introduced in this way has some additional and very useful characteristics. Namely, the sign of the obtained value $\psi$ tells us whether a hypersurface point seen from a given point of the observational plane is before or behind the observer. Despite the fact that with such a solution the assumed distance does not meet the metrics properties, this solution was assumed due to benefits resulting from it.

After the introduction of the above notions, we can describe the procedure serving to obtain the multidimensional virtual reality view. Such a view is calculated for the direction of projection $r$ set at a given moment and vectors $w, p_{1}, p_{2}$ set at a given moment specifying the location and orientation of observational plane $P=\delta\left(w,\left\{p_{1}, p_{2}\right\}\right)$. The obtained view will have the specified resolution, that is, it will comprise a finite number of pixels. For every such a pixel with coordinates $\beta_{1}, \beta_{2}$ specifying its location on observational plane $P=\delta\left(w,\left\{p_{1}, p_{2}\right\}\right)$, we will calculate its brightness. At the beginning, for every convex solid $Y$ we have to determine which face of the convex solid is the closest in accordance with direction $r$ to the pixel with coordinates $\beta_{1}, \beta_{2}$ specifying its location on observational plane $P$. The one which is the closest will be visible. We will use formula 8 to calculate the distance from relevant hypersurface $S_{\left(s_{i}, d_{i}\right)}$ corresponding to $Z_{\left(s_{i}, d_{i}\right)}$ such that convex solid $Y$ is generated by half-spaces $Z_{\left(s_{i}, d_{i}\right)}$. However, the fact that a given hypersurface is the closest is not sufficient. Additionally, it must be verified whether given vector $a$ of the hypersurface observed in accordance with direction $r$ belongs to the face of convex solid $Y$, that is, it belongs to convex solid $Y$. We can verify this by using the convex solid definition, verifying whether the analyzed vector $a$ belongs to every half-space $Z_{\left(s_{i}, d_{i}\right)}$ such that convex solid $Y$ is generated by half-spaces $Z_{\left(s_{i}, d_{i}\right)}$. Therefore, we verify the condition:

$\forall Z_{\left(s_{i}, d_{i}\right)}$ such that $Y$ is generated by $Z_{\left(s_{i}, d_{i}\right)}$ :

$\left(a-s_{i}, d_{i}\right) \geq 0$

where $i=1 \ldots k$ and convex solid $Y$ is generated by halfspaces $Z_{\left(s_{1}, d_{1}\right)}, Z_{\left(s_{2}, d_{2}\right)}, \ldots, Z_{\left(s_{k}, d_{k}\right)}$ 
For specific vector $a \in X$ at a distance of $\psi$ from the point with coordinates $\beta_{1}, \beta_{2}$ specifying the location on observational plane $P$ with taking the perspective into account, we obtain the condition:

$\forall Z_{\left(s_{i}, d_{i}\right)}$ such that $Y$ is generated by $Z_{\left(s_{i}, d_{i}\right)}$ :

$\left((1+\psi)\left(\beta_{1} p_{1}+\beta_{2} p_{2}\right)+w-\psi \xi r-s_{i}, d_{i}\right) \geq 0$

where $i=1 \ldots k$ and convex solid $Y$ is generated by halfspaces $Z_{\left(s_{1}, d_{1}\right)}, Z_{\left(s_{2}, d_{2}\right)}, \ldots, Z_{\left(s_{k}, d_{k}\right)}$.

In the way described above, we can calculate which convex solid $Y$ and which face of convex solid $Y$ is the closest in accordance with direction $r$ to the pixel with coordinates $\beta_{1}, \beta_{2}$ and thus which of them is visible. If we already know this, then using formula 7 we can calculate the brightness of this fragment of the convex solid face observed in accordance with direction $r$ from the pixel with coordinates $\beta_{1}, \beta_{2}$ specifying the location of this pixel on observational plane $P$. To achieve the additional effect consisting in that objects moving away from the observer become darker, we additionally can multiply the obtained value LUM by the coefficient dependent on the distance, e.g., by: $0.9+0.1 /(1+\psi)$. The procedure described in the form of a pseudocode is presented in Algorithm 1. The procedure described above should be invoked after every change in vectors $w, p_{1}, p_{2}, r$. The presented algorithm is the same for any number of dimensions $n$, where $n \geq 3$. Only the scalar product calculation method depends on the number of dimensions. We can use the typical formula:

$(x, y)=\sum_{i=1}^{n} x_{i} y_{i}$

where $x=\left(x_{1}, x_{2}, \ldots, x_{n}\right), y=\left(y_{1}, y_{2}, \ldots, y_{n}\right)$.

\section{Experimental setup}

In the period from October 19, 2017, to November 27, 2017, the research was performed on 97 IT students at the AGH University of Science and Technology. Every participant in the research after reading a short description concerning the meaning of keys serving to move around in the virtual multidimensional space had a possibility to choose the four-dimensional or five-dimensional labyrinth and one out of three difficulty levels. Students in total made 357 attempts to leave virtual four-dimensional and fivedimensional labyrinths with three different difficulty levels.

The four-dimensional labyrinth at the first difficulty level comprised four turns, each turn in each of four possible directions. At the second level, the same labyrinth comprised eight turns, two turns in each of four possible directions. The fourdimensional labyrinth at the third level comprised the same

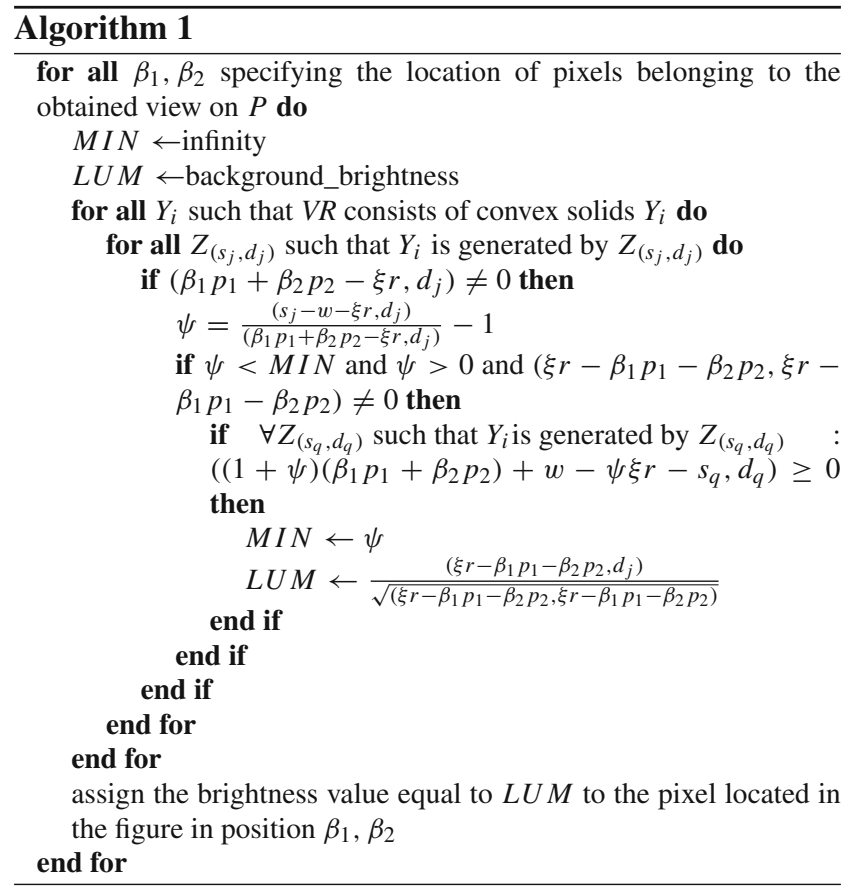

turns as the one at the second level, but additionally it had four dead ends.

Analogically, the five-dimensional labyrinth at the first difficulty level comprised five turns, each turn in each of five possible directions. At the second level, the same labyrinth comprised ten turns, two turns in each of five possible directions. The five-dimensional labyrinth at the third level comprised the same turns as the one at the second level, but additionally it had five dead ends.

The description of these labyrinths structure is more complicated. Each labyrinth comprised many sections. A section occupies the area of a regular multidimensional cube. Each section comprises walls surrounding this section from each side (apart from the entrance and potential exits from the section). The number of walls entirely limiting the section is equal to 8 for the four-dimensional section and 10 for the five-dimensional section. Each wall in the case of the fourdimensional labyrinth is a four-dimensional solid being a four-dimensional cuboid which is generated by eight halfspaces. Each wall in the case of a five-dimensional labyrinth is a five-dimensional solid being a five-dimensional cuboid which is generated by ten half-spaces. Walls have a certain thickness and remaining dimensions resulting from the section size. Each half-space is determined by two vectors. To sum up, at maximum, the section (i.e., without entrances and exits) can be determined by 128 vectors (i.e., 512 numbers) in the four-dimensional space and 200 vectors (i.e., 1000 numbers) in the five-dimensional space.

Taking the above and the fact that sections had an entrance and potential exits into account, the four-dimensional labyrinth comprised seven sections comprising 43 walls in 
total (344 half-spaces, 2752 numbers) at level 1, 11 sections comprising 67 walls in total (536 half-spaces, 4288 numbers) at level 2 and 15 sections comprising 91 walls in total (728 half-spaces, 5824 numbers) at level 3 . The five-dimensional labyrinth comprised accordingly eight sections comprising 65 walls in total (650 half-spaces, 6500 numbers) at level 1 , 13 sections comprising 105 walls in total (1050 half-spaces, 10,500 numbers) at level 2 and 18 sections comprising 145 walls in total (1450 half-spaces, 14,500 numbers) at level 3.

A computer program allowing to virtually move inside a four-dimensional and five-dimensional labyrinth was created in order to perform the research. This program was created based on the visualization method presented above. It acts as a Web page server, enabling the remote connection of many users through the Internet browser, every one of whom can independently make an attempt to leave the multidimensional labyrinth. GPU GTX1080Ti comprising 3584 cores was used in order to accelerate calculations. The calculation of one view of the four-dimensional labyrinth lasted for $0.0057(\mathrm{~s})$, while the calculation of one view of the five-dimensional labyrinth lasted for 0.0073 (s). In practice, the calculation power was sufficient for the simultaneous presence and movement of 15 people in the labyrinth. This program was created as a Web server in the $\mathrm{C}++$ programming language, and on the Web browser side, there is an application written in JavaScript to communicate with this server. The whole comprises the following procedures:

The main process This process awaits the acceptance of the incoming TCP/IP connection at port 80 classically in a loop. In the case of such a connection appearance, it creates a new thread and the connection handling procedure is launched in it, while it goes back to awaiting the next connection.

The connection handling procedure It receives data sent through the connection TCP/IP accepted earlier and interprets it in accordance with the HTTP protocol. The request type is recognized:

- If the request is the request for the page content, then the demanded page element, that is, the demanded picture or the HTML page content along with the JavaScript code, is sent in accordance with the HTTP protocol in response to this request. Then, the thread is terminated.

- If the request is a command from the application in JavaScript, then, based on the received pressed key code, the value of vectors $w, p_{1}, p_{2}, r$ is changed. If instead of the key code the request for beginning is received, then these vectors assume initial values. If the request for termination is received, then the test result is remembered and the thread is terminated only for this case at this moment. Then, with the assumed resolution, using GPU for each point of the screen, its brightness is calculated in accordance with the procedure described in section 3. The obtained view is sent in response to the applica-

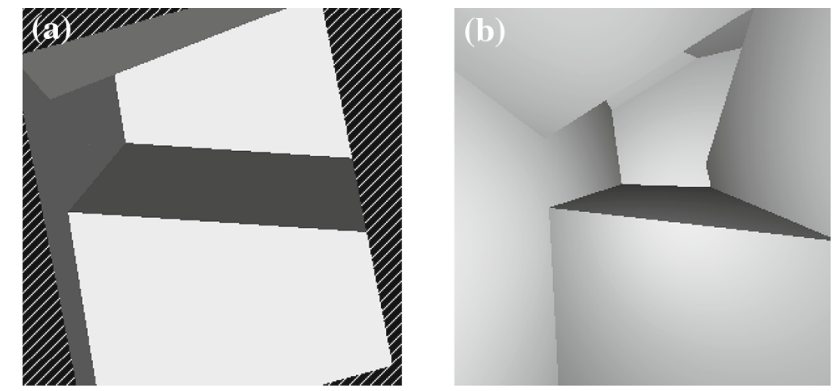

Fig. 1 Differences in the five-dimensional labyrinth interior visualization using the previous method not taking perspective into account (part a) and the MVR method taking perspective into account (part b). Both figures present the view of exactly the same labyrinth fragment

tion in JavaScript as a byte stream. Then, the thread is terminated.

The application in JavaScript on the Web browser side. In the case of pressing the key, a request to calculate the view is sent along with the code of the pressed key. In the case of pressing button Start, a request to start a test and assume initial values by vectors $w, p_{1}, p_{2}, r$ is sent. In the case of pressing the button End, a request to end is sent. In the case of accepting the data describing the obtained view from the server, it is displayed in the browser window.

\section{Results and discussion}

Figures 1, 2, 3, 4 and 5 present the obtained labyrinths' interior views. Figure 1 presents differences in the fivedimensional labyrinth interior view between the previously used method [6] and the MVR method presented in the paper which takes perspective into account. The difference is colossal, because along with the perspective we obtain the additional information on how far separate objects are from the observer. If the multidimensional object moves away from the observer, then the size of its image presented on the computer screen decreases. Figure 2 presents changes in the five-dimensional reality view as a result of the forward movement. In the previous method [6], which does not take perspective into account, there was no possibility to observe such changes which constituted a serious obstacle in the conscious and intended movement in the multidimensional space. Views obtained in the method presented in the paper are more spacious, because the depth, to which we are accustomed to in the everyday observation of the threedimensional reality, is visible in them.

Figure 3 presents six example interior views of the fourdimensional labyrinth obtained using the MVR method. It can be seen in the figure that these are views we are not accustomed to. It should be noted that these views present 
Fig. 2 Changes in the five-dimensional labyrinth interior view as a result of a virtual observer movement using the MVR method. Part a presents the initial situation, part b the observer's forward movement and part $\mathbf{c}$ the further forward movement

Fig. 3 Example obtained interior views of the four-dimensional labyrinth These views escape our experience based on the observation of only the three-dimensional space
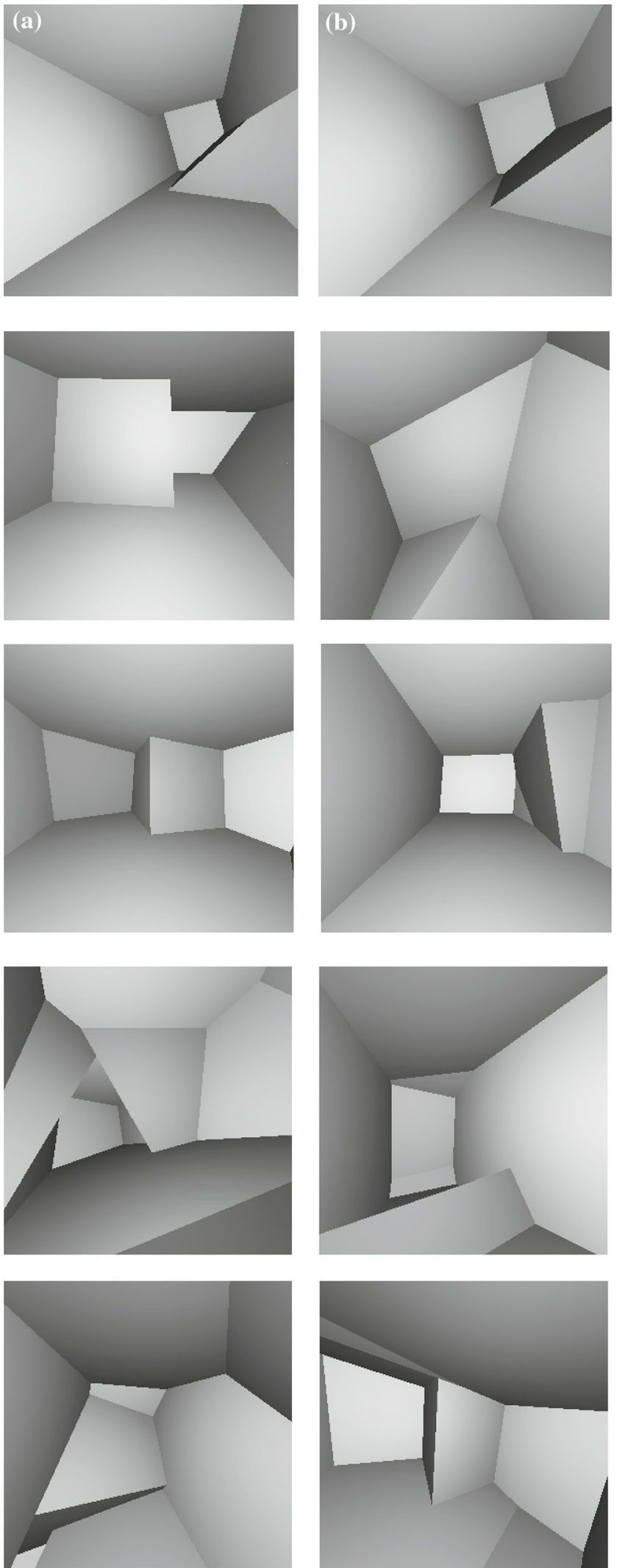
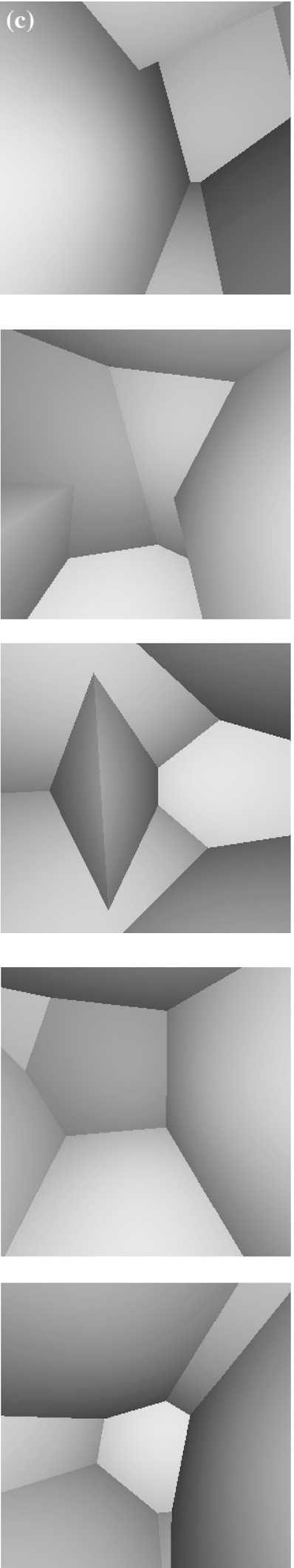
Fig. 5 Example views of the exit from the five-dimensional labyrinth. All three views present the same exit (white fragment) seen from different perspectives
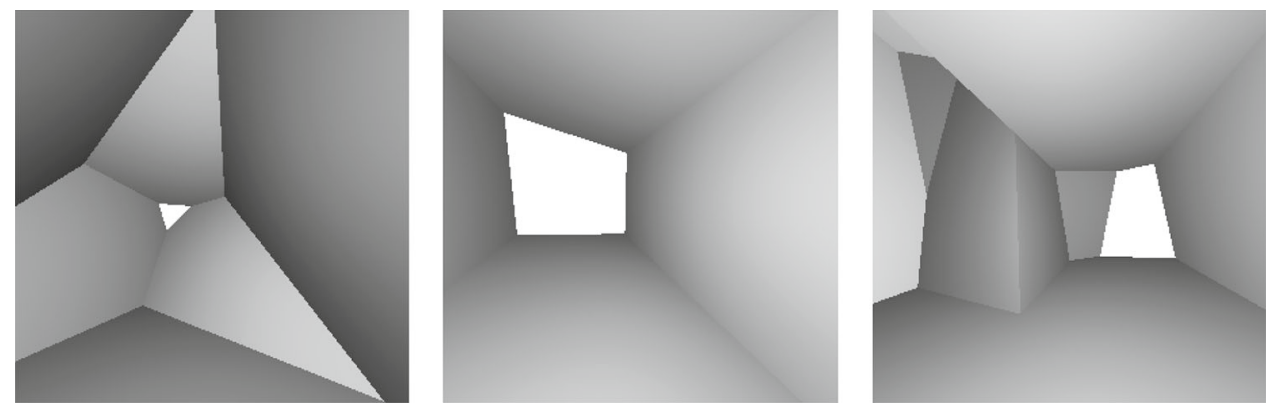

the labyrinth interior comprising fragments in the shape of regular four-dimensional cubes. However, our reception of the presented views based on the experience from the threedimensional space can be misleading. Figure 4 presents example interior views of the five-dimensional labyrinth obtained using the MVR method. This labyrinth interior comprises in turn fragments in the shape of regular fivedimensional cubes. It can be seen in the figure in an even more readable way that it is space the understanding of which and orientation in which we have to teach our brain. Figure 5 presents example views of the exit from the labyrinth.

Table 1 presents the summary of results by the effectiveness of attempts made to leave the labyrinth. It can be observed that 210 attempts to leave the four-dimensional labyrinth and 147 attempts to leave the five-dimensional labyrinth were made which make 357 attempts in total. It can also be seen that $57 \%$ attempts to leave the fourdimensional labyrinth were successful and $28 \%$ attempts to leave the five-dimensional labyrinth were successful. It follows from this that the success rate for the five-dimensional labyrinth is over two times lower. That means that leaving the five-dimensional labyrinth is significantly more difficult than leaving the four-dimensional labyrinth. The obtained result is thus a confirmation of the fact that the five-dimensional space is significantly more complicated than the four-dimensional space. It can be seen that the highest leaving success rate equal to $64 \%$ was obtained for level 3 of the four-dimensional labyrinth despite the fact that it was more difficult than levels 1 and 2. It must be noted that attempts at level 3 were usually made after making attempts at lower levels. It can be concluded that such a good result obtained for level 3 results from some kind of experience gained previously. The lowest leaving success rate equal to $11 \%$ was obtained for level 3 of the five-dimensional labyrinth, that is, for the most complicated version of the labyrinth.

Table 2 presents the summary of results by the effectiveness of people attempting to leave the labyrinth. It can be observed that all of these 97 people participating in the research made an attempt to leave the four-dimensional labyrinth at level 1 . In the case of the remaining levels and the five-dimensional labyrinth, only a part of subjects made such an attempt. It can also be seen that $70 \%$ people participating in the research managed to leave the four-dimensional labyrinth at the third difficulty level. However, only $14 \%$ people participating in the research managed to leave the five-dimensional labyrinth at the third difficulty level. It can be seen that for the four-dimensional labyrinth for all difficulty levels, the approximated percent from 67 to $73 \%$ of people participating in the research managed to leave the labyrinth. It can also be observed that for the five-dimensional labyrinth differences are greater: $38 \%$ for level 1, 30\% for level 2 and only $14 \%$ for level 3. To sum up, it turned out that about $70 \%$ people making an attempt managed to leave the four-dimensional labyrinth regardless of the difficulty level. However, for the five-dimensional labyrinth, 38\% people making an attempt managed to leave level 1,30\%—level 2 and only $14 \%$-level 3.

To move around in the multidimensional space, it was possible to use keys serving to change the direction of looking that is to make turns and move forward, backward, to the right, to the left, up and down in relation to the screen. Additionally, to make things easier, there was a possibility to use keys causing the movement directly along separate coordinate axes, that is, in four directions for the four-dimensional space and in five directions for the five-dimensional space. Table 3 presents the summary of results by the effectiveness of attempts made to leave the labyrinth without using keys serving to move along axes. By comparing this table with Table 1, you can, however, observe that there is no clear dependence between the resignation from using these keys and deterioration in the effectiveness of made attempts. It can be seen that the effectiveness deteriorated at level 3 of the four-dimensional labyrinth and at level 1 of the five-dimensional labyrinth. At the remaining levels, the effectiveness index even increased. It follows from this that the introduction of such a convenience is unnecessary.

Table 4 presents the summary of results by the duration of attempts made to leave the labyrinth. It can be noticed that the duration of presence in the labyrinth was equal to $125,212 \mathrm{~s}$, that is, over $34 \mathrm{~h}$. The average duration of the attempt to leave for all types of labyrinths was equal to $351 \mathrm{~s}$. However, the average duration of successful attempts to leave for all types of labyrinths was equal to $333 \mathrm{~s}$. The minimum duration of successful attempts to leave reached the lowest value equal to 
Table 1 The summary of results presenting the effectiveness of attempts made to leave the labyrinth

\begin{tabular}{|c|c|c|c|c|c|c|c|c|}
\hline \multicolumn{4}{|c|}{ Four-dimensional labyrinth } & \multicolumn{4}{|c|}{ Five-dimensional labyrinth } & \multirow{2}{*}{$\begin{array}{l}4 \mathrm{D}+5 \mathrm{D} \\
\text { Labs. Total }\end{array}$} \\
\hline Level 1 & Level 2 & Level 3 & Total levels $1-3$ & Level 1 & Level 2 & Level 3 & Total levels 1-3 & \\
\hline 149 & 39 & 22 & 210 & 89 & 23 & 35 & 147 & 357 \\
\hline 86 & 19 & 14 & 119 & 33 & 4 & 4 & 41 & 160 \\
\hline 58 & 49 & 64 & 57 & 37 & 17 & 11 & 28 & 45 \\
\hline
\end{tabular}

Table 2 The summary of results presenting the effectiveness of people attempting to leave the labyrinth

Four-dimensional labyrinth Five-dimensional labyrinth

Level 1 Level 2 Level 3 Level 1 Level 2 Level 3

The number of people participating in the research (making attempt to leave a labyrinth) $97 \quad \begin{array}{lllll}15 & 10 & 60 & 10 & 21\end{array}$

The number of people who were able to leave the labyrinth

$\begin{array}{lll}65 & 11 & 7\end{array}$

The percent of people who were able to leave the labyrinth (\%)

$\begin{array}{lll}67 & 73 \quad 70\end{array}$

The number of people who left the labyrinth at the first attempt

The number of people who left the labyrinth for the first time at the second attempt

The number of people who left the labyrinth for the first time at attempt $\geq 3$

$50 \quad 7 \quad 5$

$8 \quad 0 \quad 1$

$\begin{array}{lll}7 & 4 & 1\end{array}$

The average number of the successful first attempt

$\begin{array}{lll}1.38 & 1.82 & 1.43\end{array}$

$\begin{array}{lll}23 & 3 & 3\end{array}$

$\begin{array}{lll}38 & 30 \quad 14\end{array}$

The number

Table 3 The summary of results presenting the effectiveness of attempts made to leave the labyrinth without the movement along axes

\begin{tabular}{|c|c|c|c|c|c|c|c|c|c|}
\hline & \multicolumn{4}{|c|}{ Four-dimensional labyrinth } & \multicolumn{4}{|c|}{ Five-dimensional labyrinth } & \multirow{2}{*}{$\begin{array}{l}4 \mathrm{D}+5 \mathrm{D} \\
\text { Labs. total }\end{array}$} \\
\hline & Level 1 & Level 2 & Level 3 & Total levels $1-3$ & Level 1 & Level 2 & Level 3 & Total levels $1-3$ & \\
\hline $\begin{array}{l}\text { The number of attempts made to leave the } \\
\text { labyrinth without moving along axes }\end{array}$ & 42 & 15 & 13 & 70 & 28 & 6 & 8 & 42 & 112 \\
\hline $\begin{array}{l}\text { The number of successful attempts without } \\
\text { moving along axes }\end{array}$ & 28 & 11 & 7 & 46 & 8 & 2 & 2 & 12 & 58 \\
\hline $\begin{array}{l}\text { The percent of successful attempts without } \\
\text { moving along axes }\end{array}$ & 67 & 73 & 54 & 66 & 29 & 33 & 25 & 29 & 52 \\
\hline
\end{tabular}

Table 4 The summary of results presenting the duration of attempts made to leave the labyrinth

\begin{tabular}{|c|c|c|c|c|c|c|c|c|c|}
\hline & \multicolumn{4}{|c|}{ Four-dimensional labyrinth } & \multicolumn{4}{|c|}{ Five-dimensional labyrinth } & \multirow{2}{*}{$\begin{array}{l}4 \mathrm{D}+5 \mathrm{D} \\
\text { Labs. total }\end{array}$} \\
\hline & Level 1 & Level 2 & Level 3 & Total levels $1-3$ & Level 1 & Level 2 & Level 3 & Total levels 1-3 & \\
\hline $\begin{array}{l}\text { The minimum duration time of attempts to } \\
\text { leave }(\mathrm{s})\end{array}$ & 21 & 77 & 59 & 21 & 25 & 78 & 24 & 24 & 21 \\
\hline $\begin{array}{l}\text { The maximum duration time of attempts to } \\
\text { leave }(\mathrm{s})\end{array}$ & 1739 & 859 & 1750 & 1750 & 3604 & 1527 & 2866 & 3604 & 3604 \\
\hline $\begin{array}{l}\text { The total duration time of attempts to leave } \\
\text { (s) }\end{array}$ & 35,744 & 12,597 & 9460 & 57,801 & 31,124 & 14,137 & 22,150 & 67,411 & 125,212 \\
\hline $\begin{array}{l}\text { The average duration time of attempts to } \\
\text { leave }(\mathrm{s})\end{array}$ & 240 & 323 & 430 & 275 & 350 & 615 & 633 & 459 & 351 \\
\hline $\begin{array}{l}\text { The minimum duration time of successful } \\
\text { attempts to leave (s) }\end{array}$ & 29 & 94 & 86 & 29 & 41 & 481 & 569 & 41 & 29 \\
\hline $\begin{array}{l}\text { The maximum duration time of successful } \\
\text { attempts to leave (s) }\end{array}$ & 1468 & 638 & 1750 & 1750 & 3604 & 1527 & 2364 & 3604 & 3604 \\
\hline $\begin{array}{l}\text { The total duration time of successful } \\
\text { attempts to leave (s) }\end{array}$ & 17,123 & 5476 & 6714 & 29,313 & 16,063 & 3733 & 4177 & 23,973 & 53,286 \\
\hline $\begin{array}{l}\text { The average duration time of successful } \\
\text { attempts to leave (s) }\end{array}$ & 199 & 288 & 480 & 246 & 487 & 933 & 1044 & 585 & 333 \\
\hline
\end{tabular}


29 s at level 1 of the four-dimensional labyrinth. However, the minimum duration of successful attempts to leave reached the highest value equal to $569 \mathrm{~s}$ at level 3 of the five-dimensional labyrinth. The maximum duration of successful attempts to leave reached the highest value equal to $3604 \mathrm{~s}$ at level 1 of the five-dimensional labyrinth. It can also be noticed that the average duration of successful attempts to leave for separate levels increases along with the increase in level and along with the increase in the space dimension.

Based on the results presented in the paper, it can be stated that the significant part of people participating in the research was able to purposefully move around in the fourdimensional and five-dimensional space. Thus, creating for example computer games in which action takes place in the multidimensional space can be justified. Looking at the great enthusiasm of participants to the research, it can be assumed that spending time in the multidimensional space and attempts made to leave the labyrinth brought a great deal of satisfaction. Currently, work is underway on the creation of a commercial game based on the MVR method. This game is implemented on HTC VIVE and on smartphones. It should be noted that the possibility to receive the multidimensional space views through the sense of sight can be treated as a new type of stimuli transferred through the information channel available to us, humans. It will be possible to obtain more information on the effect of spending time in the multidimensional virtual reality on our human cognitive abilities as a result of further research.

\section{Conclusions}

1. The most important conclusion obtained due to the proposed method and as a result of the conducted research is that the significant part of people can purposefully move around in the four-dimensional and five-dimensional space.

2. The method of visualization introduced in the paper allowed to obtain views from the interior of the multidimensional virtual reality.

3. $57 \%$ attempts to leave the four-dimensional labyrinth were successful and 28\% attempts to leave the fivedimensional labyrinth were successful. It follows from this that the success rate in reaching the exit for the five-dimensional labyrinth is over two times lower. That means that leaving the five-dimensional labyrinth is significantly more difficult than leaving the fourdimensional labyrinth.

4. The highest leaving success rate equal to $64 \%$ was obtained for level 3 of the four-dimensional labyrinth despite the fact that it was more difficult than levels 1 and 2. It can be concluded that such a good result obtained for level 3 results from the experience gained in previous attempts to leave the labyrinth at level 1 and level 2.

5. The lowest leaving success rate equal to $11 \%$ was obtained for level 3 of the five-dimensional labyrinth, that is, for the most complicated version of the labyrinth.

6. For all three levels of the four-dimensional and fivedimensional labyrinths, there was a part of subjects able to reach the exit. About $70 \%$ people making an attempt managed to leave the four-dimensional labyrinth regardless of the difficulty level. However, for the fivedimensional labyrinth, $38 \%$ people making an attempt managed to leave level 1,30\%-level 2 and only $14 \%$ level 3.

7. The introduction of the convenience consisting in the possibility to use keys causing the movement directly along separate coordinate axes was unnecessary.

8. The average duration of successful attempts to leave for separate levels increases along with the increase in level and along with the increase in the space dimension.

\section{Compliance with ethical standards}

Conflict of interest The author declares that he has no conflict of interest.

Open Access This article is distributed under the terms of the Creative Commons Attribution 4.0 International License (http://creativecomm ons.org/licenses/by/4.0/), which permits unrestricted use, distribution, and reproduction in any medium, provided you give appropriate credit to the original author(s) and the source, provide a link to the Creative Commons license, and indicate if changes were made.

\section{References}

1. Banchoff, T.: Beyond the Third Dimension. Scientific American Library, New York (1990)

2. Carey, S.A., Burton, R.P., Campbell, D.M.: Shades of a higher dimension. Comput. Graph. World. 10(10), 93-94 (1987)

3. Dewdney, A.K.: Computer recreations: a program for rotating hyper-cubes induces four-dimensional dementia. Sci. Am. 254(4), 14-23 (1986)

4. Hanson, A., Heng, P.: Illuminating the fourth dimension. IEEE Comput. Graph. Appl. 12(4), 54-62 (1992)

5. Inselberg, A.: Parallel Coordinates: Visual Multidimensional Geometry and Its Applications. Springer, New York (2009). https:// doi.org/10.1007/978-0-387-68628-8

6. Jamroz, D.: Multidimensional labyrinth - multidimensional virtual reality. In: Cyran K.A., Kozielski S., Peters J.F., Stanczyk U., Wakulicz-Deja A. (eds) Man-Machine Interactions, Advances in Intelligent and Soft Computing, vol. 59, pp. 445-450. Springer, Berlin (2009). https://doi.org/10.1007/978-3-642-00563-3_47C

7. Jamroz, D.: The perspective-based observational tunnels method: a new method of multidimensional data visualization. Inf. Vis. 16(4), 346-360 (2017). https://doi.org/10.1177/1473871616686634

8. Jamroz, D.: The analysis of the effectiveness of the perspectivebased observational tunnels method by the example of the evalu- 
ation of possibilities to divide the multidimensional space of coal samples. In: Computational Science-ICCS 2018, Lecture Notes in Computer Science, vol. 10862, pp. 675-682. Springer, Cham (2018). https://doi.org/10.1007/978-3-319-93713-7_64

9. Jamroz, D.: Application of perspective-based observational tunnels method to visualization of multidimensional fractals. In: Artificial Intelligence and Soft Computing, ICAISC 2018, Lecture Notes in Computer Science, vol. 10842, pp. 364-375. Springer, Cham (2018). https://doi.org/10.1007/978-3-319-91262-2_33

10. Jamroz, D., Niedoba, T., Surowiak, A., Tumidajski, T., Szostek, R., Gajer, M.: Application of multi-parameter data visualization by means of multidimensional scaling to evaluate possibility of coal gasification. Arch. Min. Sci. 62(3), 445-457 (2017). https:// doi.org/10.1515/amsc-2017-0034

11. Jolliffe, I.: Principal Component Analysis, 2nd edn. Springer, New York (2002). https://doi.org/10.1007/b98835

12. Kim, S., Kwon, S., Cook, D.: Interactive visualization of hierarchical clusters using MDS and MST. Metrika 51, 39-51 (2000)

13. Niedoba, T.: Multi-parameter data visualization by means of principal component analysis (PCA) in qualitative evaluation of various coal types. Physicochem. Probl. Miner. Process. 50(2), 575-589 (2014)

14. Niedoba, T.: Application of relevance maps in multidimensional classification of coal types. Arch. Min. Sci. 60(1), 93-106 (2015)

15. Noll, M.: A computer technique for displaing n-dimensional hyperobjects. Commun. ACM 10(8), 469-473 (1967)

16. Steiner, K., Burton, R.: Hidden volumes: the 4 th dimension. Comput. Graph. World 10(2), 71-74 (1987)
17. Wang, Y.C., Zhang, Q., Lin, F., Goh, C.K., Seah, H.S.: PolarViz: a discriminating visualization and visual analytics tool for highdimensional data. Vis. Comput. (2018). https://doi.org/10.1007/ s00371-018-1558-y

Publisher's Note Springer Nature remains neutral with regard to jurisdictional claims in published maps and institutional affiliations.

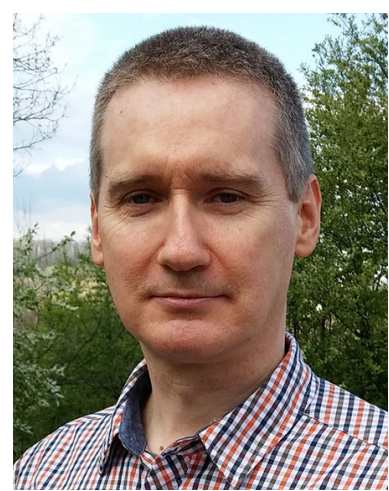

Dariusz Jamróz is adjunct professor at the Department of Applied Computer Science, AGH University of Science and Technology. He holds a $\mathrm{PhD}$ in Computer Science, and his research interests include artificial intelligence, data analysis, data mining, multidimensional visualization, information visualization and scientific visualization. 